\title{
Could the loss of diaphragm thickness measured by computer tomography predict the rate of reintubation?
}

\author{
Yue-Nan $\mathrm{Ni}^{1 \#}$, Hui Xu ${ }^{2 \#}$, Wei-Jing Li $^{1}$, Ji-Kui Sun ${ }^{3}$, Bin-Miao Liang ${ }^{1}$, Zong-An Liang ${ }^{1}$ \\ ${ }^{1}$ Department of Respiratory and Critical Care, ${ }^{2}$ Department of Radiology, West China School of Medicine and West China Hospital, Sichuan \\ University, Chengdu 610041, China; ${ }^{3}$ State Key Laboratory of Oral Diseases, West China School of Stomatology, Sichuan University, Chengdu \\ 610041, China \\ Contributions: (I) Conception and design: YN Ni, BM Liang, ZA Liang; (II) Administrative support: BM Liang, ZA Liang; (III) Provision of study \\ materials or patients: BM Liang, ZA Liang; (IV) Collection and assembly of data: YN Ni, H Xu, WJ Li, JK Sun; (V) Data analysis and interpretation: \\ YN Ni, H Xu, WJ Li, JK Sun; (VI) Manuscript writing: All authors; (VII) Final approval of manuscript: All authors. \\ "These authors contributed equally to this work. \\ Correspondence to: Zong-An Liang. No. 37 Guoxue Alley, Chengdu 610041, China. Email: liangzatg@126.com; Bin-Miao Liang. No. 37 Guoxue \\ Alley, Chengdu 610041, China. Email: liangbinmiao@163.com.
}

Background: Diaphragm function loss is very common in the intensive care unit (ICU) and can predict
the success of weaning. However, whether diaphragm thickness loss during mechanical ventilation (MV) as
measured by computed tomography (CT) can predict the rate of reintubation remains unclear. Therefore,
we hypothesized that a loss of diaphragm thickness would impact the outcome of weaning.
Methods: A retrospective study was performed on patients who received MV in the ICU of West China
Hospital, Sichuan University. The diaphragm thickness of each patient on the CT scans within 48 hours
after MV and 24 hours before weaning were measured by at least two independent investigators. The
primary outcome was the rate of reintubation, and the second outcomes included hospital mortality and the
length of ICU stay (ICU LOS) after extubation.
Results: A total of 145 patients were included in the analysis. According to the receiver operating
characteristic curve, all patients were divided into two groups (less or more than 1.55 mm diaphragm
thickness loss in reintubation). As a result, less loss of diaphragm thickness was a protective factor for the rate
of reintubation [ $33 \%$ vs. $12 \%$; adjusted odds ratio (aOR) $0.001 ; 95 \%$ confidence interval (CI), $0.001-0.271$;
P=0.018] and hospital mortality ( $18 \%$ vs. $4 \%$; a OR $0.001 ; 95 \%$ CI, 0.001-0.035; P=0.007). However, no
significant difference was found in the ICU LOS after extubation between the two groups.

Conclusions: Less diaphragm thickness loss was related to a lower rate of reintubation and hospital mortality.

Keywords: Diaphragm thickness; weaning; rate of reintubation; hospital mortality

Submitted Jul 18, 2019. Accepted for publication Dec 20, 2019.

doi: $10.21037 /$ jtd.2019.12.125

View this article at: http://dx.doi.org/10.21037/jtd.2019.12.125

\section{Introduction}

Investigations showed that approximately $2-3$ million patients in the intensive care unit (ICU) receive invasive mechanical ventilation (MV) in the United States each year $(1,2)$. Prolonged MV existed in approximately $14 \%$ of the patients who received MV (3), which would lead to many potential complications, including pulmonary barotrauma, ventilator-associated lung injury, ventilator-associated pneumonia, airway injury, venous thromboembolism, and gastrointestinal bleeding (4-8). Thus, mortality is associated with both prolonged weaning and the duration of MV $(3,9)$. Weaning is the process of decreasing ventilator support and allowing patients to use a greater proportion of their 
own ventilation. The purpose of weaning is to assess the probability that $M V$ can be successfully discontinued. Weaning may involve either an immediate shift from full ventilatory support to a period of breathing without assistance from the ventilator or a gradual reduction in the amount of ventilator support (10). However, when patients are unable to maintain spontaneous breathing, weaning could lead to reintubation and increase mortality. Thus, some variable predictors that help clinicians to estimate the capacity of patients to breathe independent of the ventilator are very important. Unfortunately, the optimal predictors that could predict the best time for weaning with high sensitivity and specificity have still not been found. Studies have shown that the current predictors, including vital capacity, tidal volume $\left(\mathrm{V}_{\mathrm{T}}\right)$, airway occlusion pressure $\left(\mathrm{P}_{0.1}\right)$, minute ventilation, respiratory rate, maximal inspiratory pressure (MIP), respiratory frequency to tidal volume ( $f / \mathrm{V}_{\mathrm{T}}$ ), $\mathrm{P}_{0.1} / \mathrm{MIP}$ and $\mathrm{P}_{0.1} \times \mathrm{f} / \mathrm{V}_{\mathrm{T}}$ are not sufficient for the prediction of weaning outcomes in a general ICU population $(11,12)$. Thus, we still need to find some new predictors to help us explore the optimal time point of weaning.

MV could lead to a loss of diaphragm thickness because of several mechanisms, including diaphragm inactivity during MV, systematic inflammation, prolonged sedation, etc. $(13-15)$. As is well known, the diaphragm is the major inspiration muscle, and it contributes largely to respiratory ability (16). Studies have shown that diaphragm function is an important determinant of successful liberation from ventilation and recovery from critical illness (17-20). Although many devices are used to measure diaphragm function, such as the electromyographic signals gathered by a nasogastric catheter, when patients receive neurally adjusted ventilatory assist ventilation, their responses are often limited because of the invasive nature of the operation and the measurements of function do not reflect the structure of the diaphragm. Recently, several studies showed that the diaphragm thickness measured by ultrasound was a new method for helping decide when and how weaning should be performed $(21,22)$. However, the low precision was a major disadvantage.

Computed tomography (CT) is a common imaging technique that is often used to evaluate the diaphragm in a static state. However, we did not find any study that assessed whether CT scans were an effective way to measure the diaphragm thickness and help clinicians predict the success of weaning. Thus, we hypothesize that CT scans could be used for measuring the thickness of the diaphragm and that a loss of diaphragm thickness was a new predictor of successful extubation from MV.

\section{Methods}

\section{Study design}

The retrospective study was conducted at the respiratory ICUs in West China Hospital, which is one of the subcenters of the National Clinical Research Center of Respiratory Disease, from December 2014 through January 2017. All study participants were included in the registration program of the National Clinical Research Center of Respiratory Disease. All patients admitted to respiratory ICUs who received $M V$ were eligible if they met the inclusion criteria and no exclusion criteria. Patients were included if they had CT scans within 48 hours of starting MV and 24 hours before weaning (Appendix 1). Patients were excluded if they were diagnosed with a neuromuscular disorder, pregnancy or were younger than 18 years of age. The study was approved by the Institutional Ethical Committee for Clinical and Biomedical Research of the First Affiliated Hospital of Guangzhou Medical University (Guangzhou, China). Informed consent was waived because this was a retrospective study.

\section{CT scan analysis}

All CT imaging of the chest was acquired using multislice CT equipment (Siemens 128 slice and Philips 128 slice) at the end of inspiration in the supine position according to the protocol of lung CT scans of the radiology department of West China Hospital, which was described in previous studies (23). All CT scans were imported from the Picture Archiving and Communication System of the radiology system and stored on a secure computer system. Scans were analyzed by syngo MultiModality Workplace Version VE40A (Siemens Software Packages) by two trained and certified investigators. They measured the dome of the right diaphragm thickness in the coronary position, which was reconstructed by a $1 \mathrm{~mm}$ thickness image between the ninth and tenth intercostal space. Each diaphragm was measured 3 times by each investigator, and the average value of the three measurements was provided by each investigator. When the difference in the diaphragm thickness of the same CT scan given by the two independent investigators was more than $10 \%$ of the average value of the two measurements, the CT scan was transferred to a third investigator. The third investigator's measurement was the final measurement.

\section{Data extraction}

For each patient, we collected information on age, gender, 
illness severity as indicated by the Acute Physiology and Chronic Health Evaluation (APACHEII) score, arterial blood gases at ICU admission, the history of smoking and alcohol abuse, diagnosis and underlying disease, the initial mode of MV and basic vital signs. Monitoring was continued until the patients were discharged from the hospital or dead. We obtained data on the rate of reintubation, hospital mortality, and the length of ICU stay (ICU LOS) after extubation. Our primary purpose was to assess the independent effect of diaphragm thickness loss regardless of other factors on reintubation.

\section{Outcomes}

The primary outcome was the rate of reintubation, and the second outcomes included hospital mortality and ICU LOS after extubation.

\section{Statistical analysis}

The study population was divided into two groups based on the overall loss of diaphragm thickness from the baseline measurement after MV to the last measurement obtained before weaning using a cutoff value that was calculated by receiver operating characteristic (ROC) analysis.

Consecutive variables were reported as the mean \pm standard deviation or median [interquartile range (IQR)], while categorical variables were reported as frequency and proportion. Student's t test, the Mann-Whitney U-test and the Kruskal-Wallis test were used for comparisons between continuous variables, and the chi-squared test or Fisher's exact test were used for comparisons between categorical variables.

To assess the relationship between the loss of diaphragm thickness and the rate of reintubation as well as hospital mortality, we performed a logistic regression model and adjusted for variables independently associated with the rate of reintubation or hospital mortality that had a $\mathrm{P}$ value less than 0.10 . Kaplan-Meier curves were plotted to assess the time from extubation to death, and the median time from extubation to death was compared by means of the log-rank test. All analyses were performed with SPSS 19.0 (SPSS Inc), and a 2-sided P value less than 0.05 was considered statistically significant.

\section{Results}

\section{Patients}

From December 2014 through January 2017, a total of
491 patients were admitted to the respiratory ICUs, and 9 patients were diagnosed with neuromuscular disease. One hundred seventy-six patients had CTs within 48 hours of MV and 24 hours before weaning for diagnostic purposes, and 31 scans were excluded because of low quality, windowing and artifacts (Figure 1) We performed ROC curve analysis for diaphragm thickness in reintubation and, according to the Youden index, all patients were divided into two groups: a group with a loss of diaphragm thickness less than $1.55 \mathrm{~mm}(\mathrm{n}=85)$ and a group with a loss of diaphragm thickness more than $1.55 \mathrm{~mm}(\mathrm{n}=60)$. The area under the ROC curve for reintubation was 0.666 and for hospital mortality was 0.752 .

\section{Characteristics at inclusion}

The characteristics of the patients at enrollment are shown in Table 1. The mean age and proportion of male patients with more than $1.55 \mathrm{~mm}$ in diaphragm thickness loss and less than $1.55 \mathrm{~mm}$ in diaphragm thickness loss were 66.53 vs. $64.71(\mathrm{P}=0.497)$ and $67 \%$ vs. $60 \%(\mathrm{P}=0.413)$, respectively. There was no significant difference in the APACHE II score at ICU admission $(19.87 \pm 6.90$ vs. $19.51 \pm 7.07, \mathrm{P}=0.760)$ between the groups. We did not find significant differences in baseline diaphragm thickness, MV length, body temperature, respiratory rate, heart rate, blood pressure or $\mathrm{pH}, \mathrm{PaCO}_{2}$ or oxygenation index (OI) at ICU admission.

\section{Rate of reintubation}

Overall, 30 of 145 patients (21\%) underwent reintubation after extubation. The characteristics of patients with reintubation are presented in Table 2. No difference was found between the two groups. In the unadjusted analysis, the rate of reintubation was higher in the group with more diaphragm thickness loss ( $33 \%$ vs. $12 \%, \mathrm{P}<0.01)$. In the logrank test, significant differences also existed in the median time from extubation to reintubation $(\mathrm{P}=0.002)$ (Figure 2). After adjusting for $\mathrm{pH}$ and $\mathrm{PaCO}_{2}$ at ICU admission before extubation (Table 2), we found that a greater loss of diaphragm thickness was associated with a higher rate of reintubation (aOR 0.001; 95\% CI, 0.001-0.271; $\mathrm{P}=0.018$ ).

\section{Hospital mortality}

Table 3 presents the characteristics of the patients who survived and died. Except for the OI at ICU admission (201.74 vs. 249.12, $\mathrm{P}=0.029$ ), we did not find any difference 


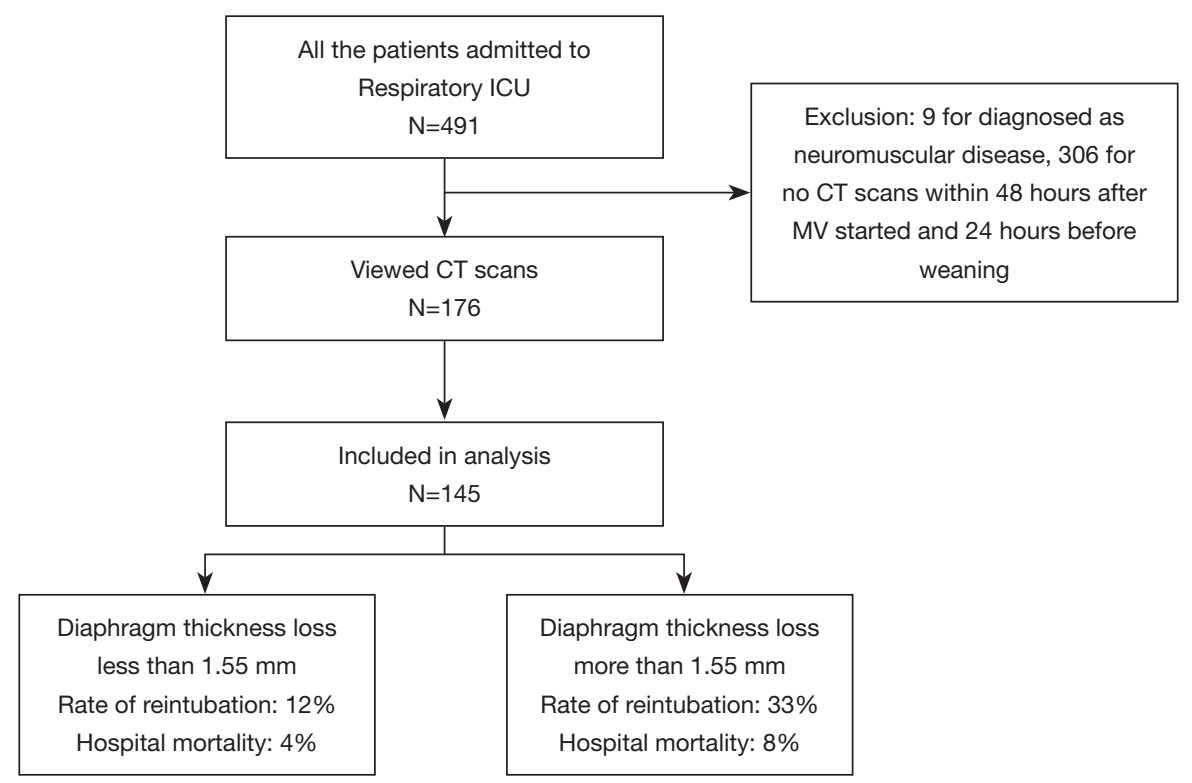

Figure 1 Flowchart of the study. CT, computed tomography; ICU, intensive care unit; MV, mechanical ventilation.

Table 1 Characteristics of patients at baseline

\begin{tabular}{|c|c|c|c|}
\hline Indexes & $\begin{array}{l}\text { More diaphragm thickness } \\
\text { loss }(>1.55 \mathrm{~mm})(\mathrm{n}=60)\end{array}$ & $\begin{array}{l}\text { Lower diaphragm thickness } \\
\text { loss }(<1.55 \mathrm{~mm})(\mathrm{n}=85)\end{array}$ & $\mathrm{P}$ \\
\hline Age, years & $66.53 \pm 14.01$ & $64.71 \pm 18.29$ & 0.497 \\
\hline APACHE II score & $19.87 \pm 6.90$ & $19.51 \pm 7.07$ & 0.760 \\
\hline Smoking & $32[53]$ & 34 [40] & 0.112 \\
\hline \multicolumn{4}{|l|}{ Diagnosis } \\
\hline Pneumonia & $23[38]$ & $41[48]$ & 0.237 \\
\hline COPD & 32 [53] & $37[44]$ & 0.206 \\
\hline PSV & $8[13]$ & $12[14]$ & \\
\hline PEEP, $\mathrm{cmH}_{2} \mathrm{O}$ & $5.37 \pm 1.63$ & $6.35 \pm 5.30$ & 0.110 \\
\hline \multicolumn{4}{|l|}{ Underlying disease } \\
\hline Diabetes & 7 [12] & $5[6]$ & 0.174 \\
\hline Hypertension & $10[17]$ & 16 [19] & 0.739 \\
\hline Arrhythmia & $4[7]$ & $6[7]$ & 0.601 \\
\hline
\end{tabular}

Table 1 (continued) 
Table 1 (continued)

\begin{tabular}{lcc}
\hline Indexes & $\begin{array}{c}\text { More diaphragm thickness } \\
\text { loss }(>1.55 \mathrm{~mm})(\mathrm{n}=60)\end{array}$ & $\begin{array}{c}\text { Lower diaphragm thickness } \\
\text { loss }(<1.55 \mathrm{~mm})(\mathrm{n}=85)\end{array}$ \\
\hline Arterial blood gas at ICU admission & $7.41 \pm 0.06$ & $7.42 \pm 0.06$ \\
$\mathrm{PH}$ & $49.50 \pm 13.93$ & $46.82 \pm 14.86$ \\
$\mathrm{PaCO}{ }_{2}, \mathrm{CmH}_{2} \mathrm{O}$ & $192.79 \pm 69.77$ & $215.86 \pm 81.53$ \\
OI & $36.71 \pm 0.69$ & $36.91 \pm 0.56$ \\
$\mathrm{~T},{ }^{\circ} \mathrm{C}$ & $20.1 \pm 8.88$ & $21.6 \pm 8.13$ \\
RR, times/hour & $96.30 \pm 25.89$ & 9.275 \\
HR, times/hour & $134.02 \pm 26.18$ & 0.077 \\
SBP, mmHg & $76.10 \pm 16.63$ & $130.39 \pm 18.95$ \\
DBP, mmHg & $9.52 \pm 1.57$ & 0.289 .96 \\
Length of ICU stay after extubation, days & $11[18]$ & 0.605 \\
Hospital mortality & $20[33]$ & 0.334 \\
Rate of reintubation & $12.18 \pm 8.92$ & 0.526 \\
MV time, days & $0.46 \pm 13.22$ \\
\hline
\end{tabular}

Data are shown as mean \pm SD or number [\%]. APACHE, The Acute Physiologic and Chronic Health Evaluation; COPD, chronic obstructive pulmonary disease; DBP, diastole blood pressure; HR, heart rate; ICU, intensive care unit; OI, oxygenation index; $\mathrm{PaCO}_{2}$, partial pressure of carbon dioxide in arterial blood; PEEP, positive end expiration pressure; PSV, pressure support ventilation; RR, respiratory rate; SBP, systolic blood pressure; T, temperature.

Table 2 Comparison between patients with reintubation and not

\begin{tabular}{|c|c|c|c|}
\hline Indexes & Non-reintubation $(n=115)$ & Reintubation $(n=30)$ & $\mathrm{P}$ \\
\hline Male & $74[64]$ & $18[60]$ & 0.660 \\
\hline APACHE II score & $20.43 \pm 6.94$ & $19.45 \pm 7.00$ & 0.495 \\
\hline Baseline diaphragm thickness, $\mathrm{mm}$ & $0.41 \pm 0.05$ & $0.41 \pm 0.07$ & 0.920 \\
\hline Change in diaphragm thickness, $\mathrm{mm}$ & $0.13 \pm 0.08$ & $0.17 \pm 0.06$ & 0.018 \\
\hline Smoking & $54[47]$ & $12[40]$ & 0.496 \\
\hline Acohol abuse & 27 [23] & $9[30]$ & 0.462 \\
\hline \multicolumn{4}{|l|}{ Diagnosis } \\
\hline Other & $10[9]$ & $2[7]$ & 0.531 \\
\hline \multicolumn{4}{|l|}{ Initial mode of mechanical ventilation } \\
\hline Control & $108[94]$ & 27 [90] & 0.342 \\
\hline PSV & $7[6]$ & $3[10]$ & \\
\hline
\end{tabular}

Table 2 (continued) 
Table 2 (continued)

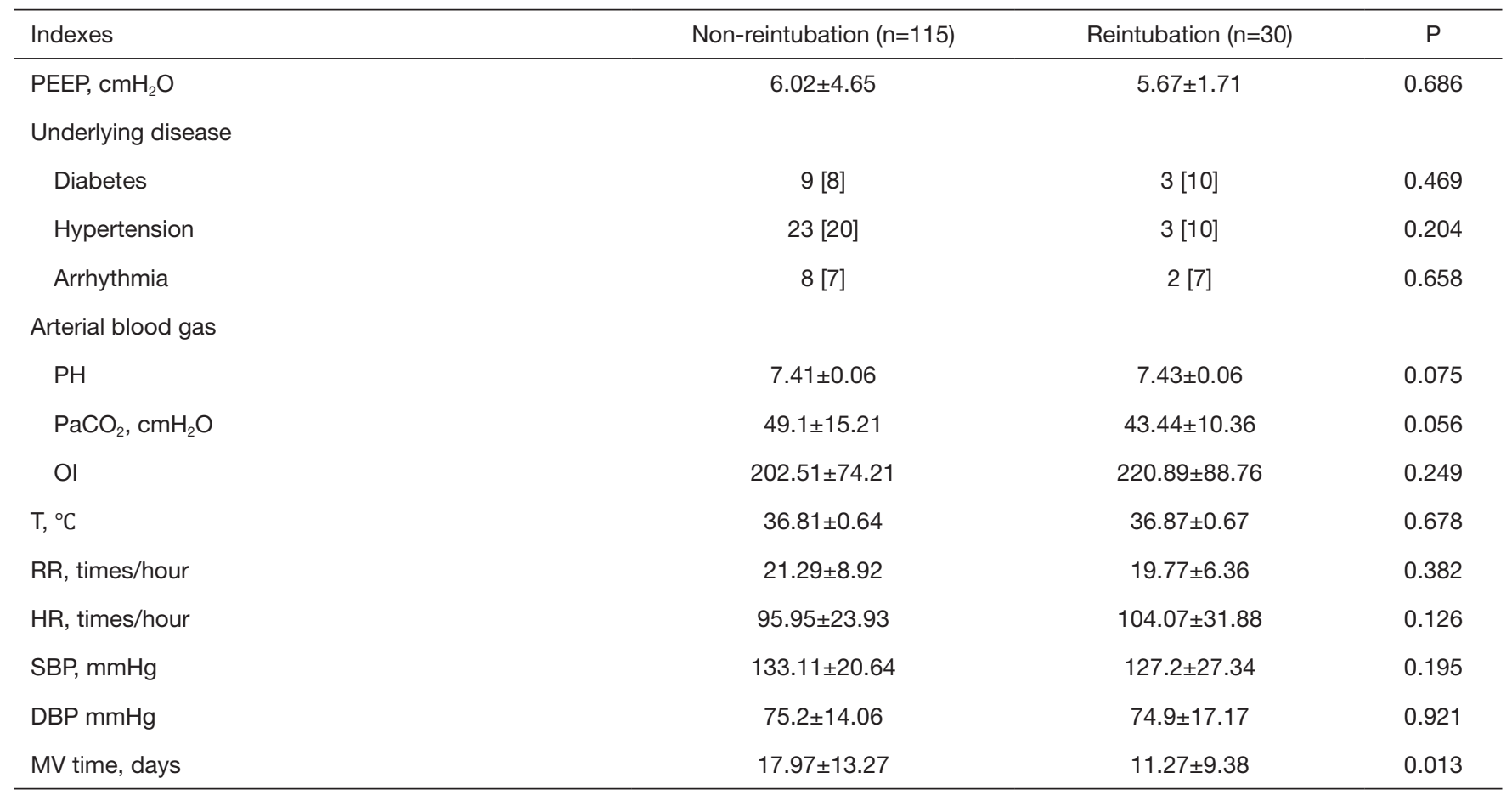

Data are shown as mean \pm SD or number [\%]. APACHE, The Acute Physiologic and Chronic Health Evaluation; COPD, chronic obstructive pulmonary disease; DBP, diastole blood pressure; $\mathrm{HR}$, heart rate; OI, oxygenation index; $\mathrm{PaCO}_{2}$, partial pressure of carbon dioxide in arterial blood; PEEP, positive end expiration pressure; PSV, pressure support ventilation; RR, respiratory rate; SBP, systolic blood pressure; $\mathrm{T}$, temperature.

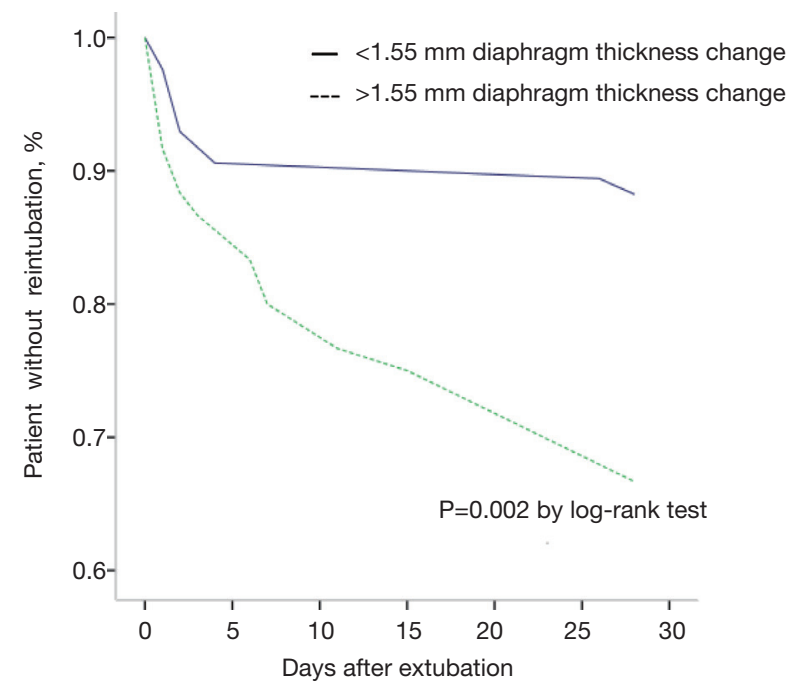

Figure 2 Kaplan-Meier curve of patients without reintubation.

between the patients that survived and died. Hospital mortality was lower in patients who had less diaphragm thickness loss $(18 \%$ vs. $4 \%, \mathrm{P}<0.01)$. After adjustment for alcohol abuse, OI and HR at ICU admission, the difference remained significant (Table 4): aOR 0.001; 95\% CI, 0.001-0.035 ( $\mathrm{P}=0.007)$. In the log-rank test, significant differences also existed in the median time from extubation to death $(\mathrm{P}=0.004)$ (Figure 3).

\section{ICU LOS after extubation}

We did not find any significant difference between the patients with more than and those with less than $1.55 \mathrm{~mm}$ diaphragm thickness loss in terms of ICU LOS (9.52 vs. 9.47 days, $\mathrm{P}=0.98)$.

\section{Discussion}

In this study, all patients who received MV had diaphragm thickness loss. In addition, less loss of diaphragm thickness between the baseline measurement after MV and the last measurement obtained before weaning was related to a lower rate of reintubation and hospital mortality.

Our study showed that the higher the diaphragm 
Table 3 Comparison between patients survival and death

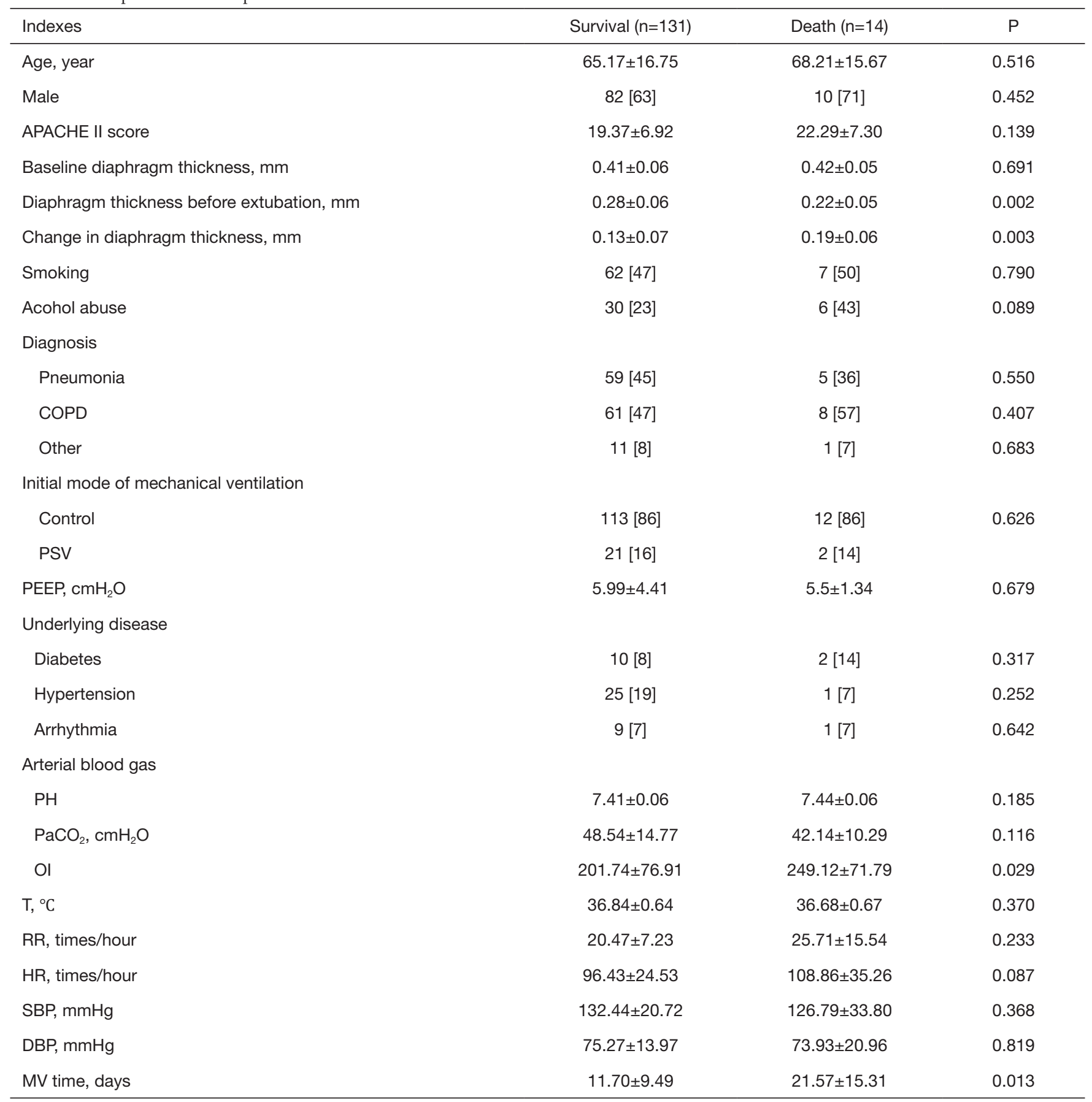

Data are shown as mean \pm SD or number [\%]. APACHE, The Acute Physiologic and Chronic Health Evaluation; COPD, chronic obstructive pulmonary disease; DBP, diastole blood pressure; HR, heart rate; OI, oxygenation index; $\mathrm{PaCO}_{2}$, partial pressure of carbon dioxide in arterial blood; PEEP, positive end expiration pressure; PSV, pressure support ventilation; RR, respiratory rate; SBP, systolic blood pressure; $\mathrm{T}$, temperature. 
Table 4 Effect of diaphragm thickness change on clinical outcomes of patients

\begin{tabular}{lcccc}
\hline \multirow{2}{*}{ Indexes } & \multicolumn{1}{c}{ Unadjusted OR } & & Adjusted OR \\
\cline { 2 - 3 } \cline { 4 - 5 } Reintubation & Odds ratio $(95 \% \mathrm{Cl})$ & P value & & Odds ratio (95\% Cl) \\
Hospital mortality & $0.267(0.114-0.624)$ & 0.002 & $0.001(0.001-0.271)$ & 0.018 \\
\hline
\end{tabular}

$\mathrm{Cl}$, confidence interval; OR, odds ratio.

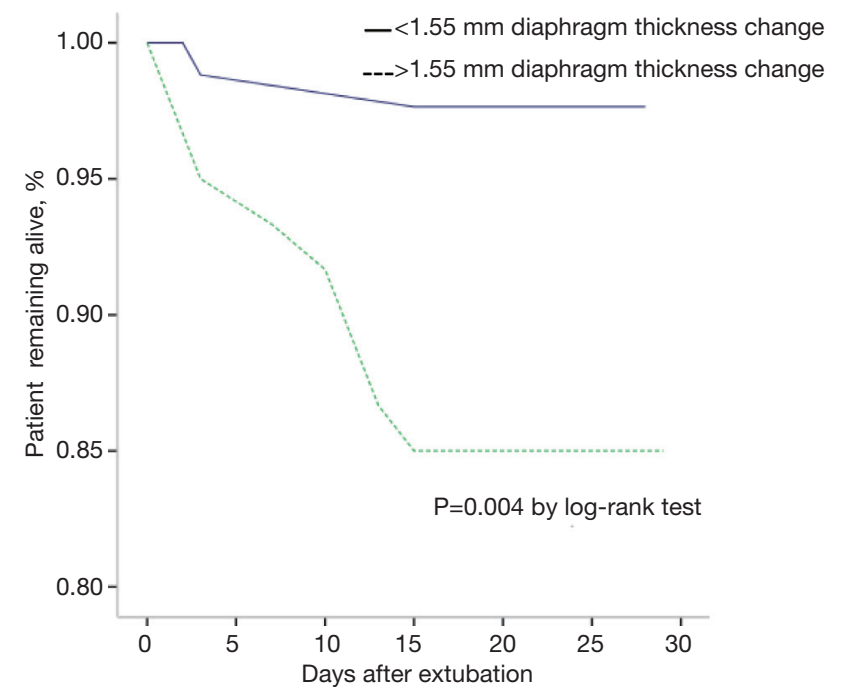

Figure 3 Kaplan-Meier curve of patients who survived.

thickness loss, the higher the rate of reintubation. According to previous findings, the decrease in diaphragm thickness during $M V$ could be explained by the following. First, MV itself could lead to diaphragm function loss. It has been shown that MV might cause diaphragmatic dysfunction in animal models (24). The main mechanism was that MV could cause a significant decrease in diaphragm force and fiber dimensions and an increase in lipid accumulation $(25,26)$. Recently, some other studies showed that decreases in diaphragm thickness are common during MV in humans, which suggests impaired diaphragmatic function (27). A study performed by Grosus showed that the overall rate of decrease in the diaphragm thickness of patients over time was an average of $6 \%$ per day of MV (28). Second, inflammation was very common in critical patients and was another factor that contributed to diaphragm dysfunction and thickness loss. Apart from atrophy resulting from systematic inflammation, increased pro-inflammatory cytokine production and autophagy, a worsening of oxidative stress and increasing nitric oxide levels may be the major factors that lead to diaphragm thickness loss $(29,30)$.
Third, prolonged sedation also had an impact on diaphragm thickness. Research has shown that sedation inhibits respiratory drive and causes diaphragm immobilization (31). A study of 36 patients found that diaphragmatic strength decreased during sedation, which was measured by ultrasound (32). Fourth, systemic muscular weakness and neuromuscular weakness are common among patients who undergo MV because of immobilization, the use of neuromuscular blocking agents, and critical illness (33). Fifth, malnutrition also contributes to the loss of thickness of the diaphragm (34) and is very common in critically ill patients (35). Moreover, studies showed that in patients with chronic hyperinflation states, diaphragm muscle length shortened by $30 \%$ to $40 \%$ from residual volume to total lung capacity (36). Autopositive end expiratory pressure (auto-PEEP) can influence both respiratory and cardiac function by causing alveolar overdistension and can lead to hypoxemia if V/Q mismatch increases due to compression of the adjacent pulmonary blood vessels. Auto-PEEP and hyperinflation also increase intrathoracic pressure, which can decrease venous return, reduce cardiac output, and potentially cause hypotension $(37,38)$. Thus, the thickness of the diaphragm, which is related to the extent of auto-PEEP and dynamic hyperinflation, affects the weaning results. The diaphragm is the major inspiration muscle and contributes to the majority of volume change during inspiration (16). Additionally, a sufficient contractile diaphragm should be a prerequisite for successful extubation. Thus, a large loss of diaphragm thickness means that the patients cannot receive enough ventilation and will eventually require reintubation.

We also found higher mortality in patients with higher diaphragm thickness loss. This may be mainly due to the higher rate of reintubation. Studies have shown that many adverse events caused by reintubation are related to invasive $\mathrm{MV}$, such as ventilator-associated lung injury, ventilatorassociated pneumonia and barotrauma, which multiply the rate of mortality $(9,39)$.

However, we did not find any significant difference in ICU LOS between the two groups. It is undeniable that 
medical resources as well as expenditures are tightly related to disease outcomes, such as bed availability in general wards and insurance status. More studies that focus on this issue are necessary to draw a definite conclusion.

A study showed that in patients receiving mechanical ventilation for more than 7 days, systematic corticosteroids lead to neuromyopathy (32). Animal studies have indicated that systematic methylprednisolone progressively decreases the diaphragm force, and high doses play a more significant role than low doses (40). In our study, we found that the patients who has ever used the corticosteroids did have more diaphragm thickness loss (0.15 vs. $0.13, \mathrm{P}=0.038$, Table S1), although we did not control the dose nor length of corticosteroids usage. Further studies need to be done to find out the exact role of corticosteroids in diaphragm thickness change of patients received mechanical ventilation.

Our study has several limitations. First, it is unknown whether diaphragm thickness measured by CT scan at a single point time is representative of the changes that occur over the course of progressive disease. Second, a loss of diaphragm thickness does not imply a loss of diaphragm function, limiting the application of this measurement. Third, the thickness of the diaphragm is influenced by many factors, such as the interaction of diaphragm contour thickness, force generation, and coupling to the abdominal and thoracic musculature.

\section{Conclusions}

The patients with higher diaphragm thickness loss had a higher rate of reintubation and hospital mortality, but a significant difference in ICU LOS after extubation was not found.

\section{Acknowledgments}

We thank Professor Dongtao Lin (College of Foreign Languages, Sichuan University), who specializes in biomedical writing and editing, for copyediting this manuscript.

Funding: This study was partly supported by the National Key Research and Development Program of China (2016YFC1304303) and the Ministry of Science and Technology of the People's Republic of China (2013BAI09B09).

\section{Footnote}

Conflicts of Interest: The authors have no conflicts of interest to declare.
Ethical Statement: The authors are accountable for all aspects of the work in ensuring that questions related to the accuracy or integrity of any part of the work are appropriately investigated and resolved. Our study was approved by the Institutional Ethical Committee for Clinical and Biomedical Research of West China Hospital (Sichuan, China). Informed consent was waived because this was a retrospective study.

Open Access Statement: This is an Open Access article distributed in accordance with the Creative Commons Attribution-NonCommercial-NoDerivs 4.0 International License (CC BY-NC-ND 4.0), which permits the noncommercial replication and distribution of the article with the strict proviso that no changes or edits are made and the original work is properly cited (including links to both the formal publication through the relevant DOI and the license). See: https://creativecommons.org/licenses/by-nc-nd/4.0/.

\section{References}

1. Wunsch H, Linde-Zwirble WT, Angus DC, et al. The epidemiology of mechanical ventilation use in the United States. Crit Care Med 2010;38:1947-53.

2. Adhikari NK, Fowler RA, Bhagwanjee S, et al. Critical care and the global burden of critical illness in adults. Lancet 2010;376:1339-46.

3. Funk GC, Anders S, Breyer MK, et al. Incidence and outcome of weaning from mechanical ventilation according to new categories. Eur Respir J 2010;35:88-94.

4. Cook DJ, Fuller HD, Guyatt GH, et al. Risk factors for gastrointestinal bleeding in critically ill patients. Canadian Critical Care Trials Group. N Engl J Med 1994;330:377-81.

5. Brower RG, Lanken PN, MacIntyre N, et al. Higher versus lower positive end-expiratory pressures in patients with the acute respiratory distress syndrome. $\mathrm{N}$ Engl J Med 2004;351:327-36.

6. Acute Respiratory Distress Syndrome Network, Brower RG, Matthay MA, et al. Ventilation with lower tidal volumes as compared with traditional tidal volumes for acute lung injury and the acute respiratory distress syndrome. N Engl J Med 2000;342:1301-8.

7. Anzueto A, Frutos-Vivar F, Esteban A, et al. Incidence, risk factors and outcome of barotrauma in mechanically ventilated patients. Intensive Care Med 2004;30:612-9.

8. International consensus conferences in intensive care medicine: Ventilator-associated Lung Injury in ARDS. 
This official conference report was cosponsored by the American Thoracic Society, The European Society of Intensive Care Medicine, and The Societé de Réanimation de Langue Française, and was approved by the ATS Board of Directors, July 1999. Am J Respir Crit Care Med 1999; 160:2118-24.

9. Esteban A, Anzueto A, Frutos F, et al. Characteristics and outcomes in adult patients receiving mechanical ventilation: a 28-day international study. JAMA 2002;287:345-55.

10. Slutsky AS. Mechanical ventilation. American College of Chest Physicians' Consensus Conference. Chest 1993;104:1833-59.

11. Yang KL, Tobin MJ. A prospective study of indexes predicting the outcome of trials of weaning from mechanical ventilation. N Engl J Med 1991;324:1445-50.

12. Conti G, Montini L, Pennisi MA, et al. A prospective, blinded evaluation of indexes proposed to predict weaning from mechanical ventilation. Intensive Care Med 2004;30:830-6.

13. Levine S, Nguyen T, Taylor N, et al. Rapid disuse atrophy of diaphragm fibers in mechanically ventilated humans. $\mathrm{N}$ Engl J Med 2008;358:1327-35.

14. Jaber S, Petrof BJ, Jung B, et al. Rapidly progressive diaphragmatic weakness and injury during mechanical ventilation in humans. Am J Respir Crit Care Med 2011;183:364-71.

15. Picard M, Jung B, Liang F, et al. Mitochondrial dysfunction and lipid accumulation in the human diaphragm during mechanical ventilation. Am J Respir Crit Care Med 2012;186:1140-9.

16. Roussos C, Macklem PT. The respiratory muscles. N Engl J Med 1982;307:786-97.

17. Vassilakopoulos T, Zakynthinos S, Roussos C. The tension-time index and the frequency/tidal volume ratio are the major pathophysiologic determinants of weaning failure and success. Am J Respir Crit Care Med 1998;158:378-85.

18. Kim WY, Suh HJ, Hong SB, et al. Diaphragm dysfunction assessed by ultrasonography: influence on weaning from mechanical ventilation. Crit Care Med 2011;39:2627-30.

19. Adler D, Dupuis-Lozeron E, Richard JC, et al. Does inspiratory muscle dysfunction predict readmission after intensive care unit discharge? Am J Respir Crit Care Med 2014;190:347-50.

20. Demoule A, Jung B, Prodanovic H, et al. Diaphragm dysfunction on admission to the intensive care unit. Prevalence, risk factors, and prognostic impact-a prospective study. Am J Respir Crit Care Med 2013;188:213-9.

21. Goligher EC, Laghi F, Detsky ME, et al. Measuring diaphragm thickness with ultrasound in mechanically ventilated patients: feasibility, reproducibility and validity. Intensive Care Med 2015;41:642-9.

22. Arakawa H, Webb WR. Air trapping on expiratory highresolution CT scans in the absence of inspiratory scan abnormalities: correlation with pulmonary function tests and differential diagnosis. AJR Am J Roentgenol 1998;170:1349-53.

23. Powers SK, Shanely RA, Coombes JS, et al. Mechanical ventilation results in progressive contractile dysfunction in the diaphragm. J Appl Physiol (1985) 2002;92:1851-8.

24. Gayan-Ramirez G, de Paepe K, Cadot P, et al. Detrimental effects of short-term mechanical ventilation on diaphragm function and IGF-I mRNA in rats. Intensive Care Med 2003;29:825-33.

25. Sassoon CS, Caiozzo VJ, Manka A, et al. Altered diaphragm contractile properties with controlled mechanical ventilation. J Appl Physiol (1985) 2002;92:2585-95.

26. Goligher EC, Fan E, Herridge MS, et al. Evolution of Diaphragm Thickness during Mechanical Ventilation. Impact of Inspiratory Effort. Am J Respir Crit Care Med 2015;192:1080-8.

27. Grosu HB, Lee YI, Lee J, et al. Diaphragm muscle thinning in patients who are mechanically ventilated. Chest 2012;142:1455-60.

28. Maes K, Stamiris A, Thomas D, et al. Effects of controlled mechanical ventilation on sepsis-induced diaphragm dysfunction in rats. Crit Care Med 2014;42:e772-82.

29. Ebihara S, Hussain SN, Danialou G, et al. Mechanical ventilation protects against diaphragm injury in sepsis: interaction of oxidative and mechanical stresses. Am J Respir Crit Care Med 2002;165:221-8.

30. Vaschetto R, Cammarota G, Colombo D, et al. Effects of propofol on patient-ventilator synchrony and interaction during pressure support ventilation and neurally adjusted ventilatory assist. Crit Care Med 2014;42:74-82.

31. Rocco M, Maggi L, Ranieri G, et al. Propofol sedation reduces diaphragm activity in spontaneously breathing patients: ultrasound assessment. Minerva Anestesiol 2017;83:266-273.

32. De Jonghe B, Sharshar T, Lefaucheur JP, et al. Paresis acquired in the intensive care unit: a prospective multicenter study. JAMA 2002;288:2859-67.

33. Lands L, Desmond KJ, Demizio D, et al. The effects of 
nutritional status and hyperinflation on respiratory muscle strength in children and young adults. Am Rev Respir Dis 1990;141:1506-9.

34. McWhirter JP, Pennington CR. Incidence and recognition of malnutrition in hospital. BMJ 1994;308:945-8.

35. Antonelli M, Conti G, Bufi M, et al. Noninvasive ventilation for treatment of acute respiratory failure in patients undergoing solid organ transplantation: a randomized trial. JAMA 2000;283:235-41.

36. De Troyer A. Effect of hyperinflation on the diaphragm. Eur Respir J 1997;10:708-13.

37. Qvist J, Pontoppidan H, Wilson RS, et al. Hemodynamic

Cite this article as: Ni YN, Xu H, Li WJ, Sun JK, Liang BM, Liang ZA. Could the loss of diaphragm thickness measured by computer tomography predict the rate of reintubation? J Thorac Dis 2020;12(3):581-591. doi: 10.21037/jtd.2019.12.125 responses to mechanical ventilation with PEEP: the effect of hypervolemia. Anesthesiology 1975;42:45-55.

38. Fougères E, Teboul JL, Richard C, et al. Hemodynamic impact of a positive end-expiratory pressure setting in acute respiratory distress syndrome: importance of the volume status. Crit Care Med 2010;38:802-7.

39. Kollef MH. What is ventilator-associated pneumonia and why is it important? Respir Care 2005;50:714-21; discussion 721-4.

40. Sassoon CS, Zhu E, Pham HT, et al. Acute effects of highdose methylprednisolone on diaphragm muscle function. Muscle Nerve 2008;38:1161-72. 


\section{Appendix 1 Weaning and reintubation protocol}

\section{Weaning protocol}

The weaning protocol included daily screening for weaning readiness according to the following criteria (41):

(I) Recovery from the precipitating illness;

(II) Respiratory criteria $\left[\mathrm{PaO}_{2}: \mathrm{FiO}_{2}\right.$ (partial pressure of oxygen, arterial:fraction of inspired oxygen) ratio $>150$ with $\mathrm{FiO}_{2} \leq 0.4$, $\mathrm{PEEP}<8 \mathrm{cmH}_{2} \mathrm{O}$, and arterial $\mathrm{pH}>7.35$ ];

(III) Clinical criteria(absence of electrocardiographic signs of myocardial ischemia, no vasoactive drugs, or only low doses of dopamine ( $<5 \mu \mathrm{g} / \mathrm{kg} / \mathrm{min})$, heart rate $<140 / \mathrm{min}$, hemoglobin $>8 \mathrm{~g} / \mathrm{dL}$, temperature $<38^{\circ} \mathrm{C}$, no need for sedatives, presence of respiratory stimulus, and appropriate spontaneous cough).

(IV) Patients fulfilling these criteria underwent a spontaneous breathing trial with pressure support for 30 to 120 minutes.

(V) Patients who tolerated the spontaneous breathing trial were reconnected with the previous ventilator settings for rest and clinical evaluation of airway patency, respiratory secretions, and upperairway obstruction.

Standard of reintubation patients were reintubated for persistent postextubation respiratory failure if they met at least one of the following criteria:

(I) Lack of improvement in $\mathrm{pH}$ or in the partial pressure of carbon dioxide or fall in GCS score $>2$ points.

(II) Lack of improvement in signs suggestive of respiratory-muscle fatigue or worsening including the appearance of unequivocal signs of respiratorymuscle fatigue, such as maintained active contraction of the expiratory muscles, asynchronous motion of the rib cage and abdomen, respiratory alternans, or active contraction of the sternocleidomastoid.

(III) Hypotension, with a systolic blood pressure below $90 \mathrm{mmHg}$ for more than 30 minutes despite adequate volume challenge, use of vasopressors, or both.

(IV) Copious secretions that could not be adequately cleared or that were associated with acidosis, hypoxemia, and changes in mental status or persistent or worsening signs of respiratory-muscle fatigue.

(V) Decrease to $\mathrm{SpO}_{2}<85 \%$ despite the use of a high $\mathrm{FiO}_{2}(>0.5)$.

Patients fulfilling these criteria were reintubated, but the final decision to reintubate was made by the treating physician or evaluated by a consensus committee excluding the investigators. The single most relevant reason for reintubation from the list was recorded. If two or more criteria were present, the reason for reintubation was assigned in the following order of preference: presence of copious secretions, respiratory acidosis, hypoxemia, signs of respiratory-muscle fatigue, and hypotension for selection of cause of reintubation.

\section{References}

41. Boles JM, Bion J, Connors A, et al. Weaning from mechanical ventilation. Eur Respir J 2007;29:1033-56.

Table S1 The effect of corticosteroids on diaphragm thickness loss

\begin{tabular}{lccc}
\hline & Non-corticosteroids $(n=80)$ & Corticosteroids $(n=65)$ & $P$ \\
\hline Diaphragm thickness loss & $0.13 \pm 0.08$ & $0.15 \pm 0.07$ & 0.038 \\
\hline
\end{tabular}

